\title{
Fidelity and purity of quantum electrical circuit states and quantum tomograms
}

\author{
Olga V. Man'ko \\ P. N. Lebedev Physical Institute, Leninskii Prospect 53, Moscow 119991, Russia \\ E-mail: omanko@sci.lebedev.ru
}

\begin{abstract}
Review of the probability representation of quantum mechanics and the symplectic tomography approach are presented. The examples of Gaussian states of nanoelectric circuit, Josephson junction, and two interacting high-quality resonant circuits are considered. The Shannon entropy, quantum information, fidelity, and purity of quantum states in the tomographic representation of quantum mechanics are studied.

PACS numbers: 03.65.-w quantum mechanics, 03.67.-a quantum information, 03.65, $\mathrm{Wj}$ quantum tomography
\end{abstract}

\section{Introduction}

Recently the theoretical aspects of the dynamical Casimir effect were intensively studied (see, e.g. [1 3]. The experimental results on the dynamical Casimir effect were obtained in [4]. The important ingredient of this investigation is the consideration of the effects related to the behaviour of nonstationary quantum oscillators. The oscillators can be realized by electric circuits or Josephson junctions with time-varying parameters [5-10]. The squeezing and other quantum properties of such devices were discussed in [5, 6, 8, 11 13. The quantum states of the devices can be associated with tomographic probability distributions (tomograms) considered for the oscillators, e.g., in [14,15. Since the quantum tomograms are the fair probability distributions, one can introduce such characteristics of the quantum states as tomographic Shannon entropy and information (see e.g. [16-18]).

The aim of this work is to consider the application of the tomographic approach to the problem of circuits and Josephson junctions. We obtain an explicit form of the ground state tomogram for two interacting high-quality resonant circuits as well as their tomographic entropy and the Shannon information expressed in terms of the quantum tomograms. For the Josephson junction modeled by a resonant circuit, we obtain the 
tomogram of a ground-like Gaussian state excited due to the time dependence of the critical current.

The paper is organized as follows.

In the first section, we discuss a quantum resonant circuit in the probability representation of quantum mechanics. In the second section, we consider the Josephson junction modeled by resonant circuit with time-dependent parameters, discuss the quantum tomogram of its Gaussian state and an analog of the nonstationary Casimir effect in this system. In the third section, we discuss two high-quality interacting resonant circuits and obtain the symplectic tomogram of their Gaussian state.

\section{Quantum resonant circuit}

Following [5, 19], we present a short review of high-quality resonant circuits with inductance $L$ and capacity $C$ in the tomographic-probability representation of quantum mechanics. The resonant circuit must be considered as the quantum one, if the energy of thermal fluctuations is smaller then the energy of vibration quanta $\hbar \omega>k T$, where $T$ is the temperature and $k$ is the Boltzmann constant. For example, if the wavelength is $\sim 1 \mathrm{~cm}$ and the temperature is lower than $1.4 \mathrm{~K}$, the resonant circuit must be considered as a quantum object. The Hamiltonian of the resonant circuit is of the form (see, e.g., [5])

$$
\hat{H}=\frac{1}{2}\left(\frac{\hat{Q}^{2}}{C}+L \hat{I}^{2}\right)
$$

where $\hat{Q}$ is an operator of charge on the plates of a capacitor and $\hat{I}$ is an operator of the current in the circuit. The commutation relation for the operators of charge and current is of the form

$$
[\hat{I}, \hat{Q}]=i \hbar / L
$$

For an operator of voltage on the plates of the capacitor $\hat{V}=\hat{Q} / C$, one has the commutation relation with operator of current $[\hat{I}, \hat{V}]=i \hbar \omega^{2}$, where $\omega=(L C)^{-1 / 2}$ is the plasma frequency.

In [15], the probability representation of quantum mechanics was introduced and applied for considering the photon states. It was shown that the quantum state can be determined by the symplectic tomogram, which is expressed through the Wigner function of the state. Applying usual symplectic-tomography-scheme procedure [15] to the problem of quantum circuit and following [19], we consider the general linear combination of the current and voltage

$$
\hat{J}=\frac{\mu \hat{I}}{i_{0}}+\frac{\nu \hat{V}}{u_{0}}
$$


where $\hat{I}$ is the operator of current, $\hat{V}$ is the operator of voltage, $\mu$ and $\nu$ are real numbers, which label the reference frame in the current-voltage space $(I, V)$ of the system, $i_{0}=\sqrt{\hbar \omega / L}$ and $u_{0}=\sqrt{\hbar / \omega L C^{2}}$ are the amplitudes of vacuum fluctuations of current and voltage. Thus, in our approach, the current and voltage are considered as analogs of the photon quadrature components. The linear combination (3) is an analog of the photon homodyne quadrature. We assume that the current and voltage are dimensionless ones. The state of the resonant circuit can be determined by the symplectic tomogram, which is a function of the observable $J$ and additional variables $\mu$ and $\nu$ and is expressed through the Wigner function [20] as follows [19]:

$$
w(J, \mu, \nu)=\frac{1}{2 \pi} \int W(I, V) \delta(J-\mu I-\nu V) d I d V .
$$

The symplectic tomogram is normalized and nonnegative and has all the properties of the standard probability distribution function. For example, the tomogram of the resonantcircuit ground state is given by normal distribution of the variable $J$ with the dispersion determined by the parameters $\mu$ and $\nu$,

$$
w_{0}(J, \mu, \nu)=\frac{1}{\sqrt{\pi\left(\mu^{2}+\nu^{2}\right)}} \exp \left(-\frac{J^{2}}{\mu^{2}+\nu^{2}}\right) .
$$

The information contained in tomogram $w(J, \mu, \nu)$ is overcomplete. For $\mu=\cos \theta$ and $\nu=\sin \theta$, where $\theta$ is the rotation angle of the axis in the $(I, V)$ plane, the symplectic tomogram is analogous to the optical tomogram introduced in [21] and measured in [22] where the experiment on reconstructing the photon Wigner function was performed.

The Wigner function can be reconstructed from the symplectic tomogram, in view of the inverse Radon transform,

$$
W(I, V)=\frac{1}{2 \pi} \int w(J, \mu, \nu) \exp [-i(\mu I+\nu V-J)] d \mu d \nu d J .
$$

The density operator also can be reconstructed from the symplectic tomogram

$$
\hat{\rho}=\frac{1}{2 \pi} \int w(J, \mu, \nu) e^{i(J-\mu \hat{I}-\nu \hat{V})} d J d \mu d \nu .
$$

Here we adopted the formula employed in quantum optics [23]. Due to the fact that the symplectic tomogram is the probability distribution function, one can introduce entropy and information following the Shannon prescription. The entropies associated with different types of tomograms were discussed in [16, 17, 24, 27] and were named the probability-representation entropies. The tomographic entropy, associated with the resonant-circuit state and determined by the symplectic tomogram, reads

$$
S(\mu, \nu)=-\int w(J, \mu, \nu) \ln w(J, \mu, \nu) d J .
$$


The tomographic entropy is a function of additional parameters which label a reference frame in the $(I, V)$ space.

Such known quantities as the fidelity and purity also can be calculated for the resonant-circuit state, in view of the state symplectic tomogram following [28]. Using the connection of the density operator of the resonant-circuit state and its symplectic tomogram, we obtain for the fidelity

$$
\begin{aligned}
\mathrm{F}=\operatorname{Tr}\left(\hat{\rho}_{1} \hat{\rho}_{2}\right)= & \frac{1}{2 \pi} \int w_{1}\left(J_{1}, \mu_{1}, \nu_{1}\right) w_{2}\left(J_{2},-\mu_{1},-\nu_{1}\right) \\
& \times \exp \left(i\left(J_{1}+J_{2}\right)\right) d J_{1} d J_{2} d \mu_{1} d \mu_{2} d \nu_{1} d \nu_{2},
\end{aligned}
$$

where $\hat{\rho}_{1}$ and $\hat{\rho}_{2}$ are the density operators and $w_{1}\left(J_{1}, \mu_{1}, \nu_{1}\right)$ and $w_{2}\left(J_{2},-\mu_{1},-\nu_{1}\right)$ are the tomograms associated with the first and second states of the resonant circuit, respectively.

The purity of the resonant-circuit state, which we denote $\mathrm{P}$, reads

$\mathrm{P}=\operatorname{Tr}\left(\hat{\rho}^{2}\right)=\frac{1}{2 \pi} \int w\left(J_{1}, \mu_{1}, \nu_{1}\right) w\left(J_{2},-\mu_{1},-\nu_{1}\right) \exp \left[i\left(J_{1}+J_{2}\right)\right] d J_{1} d J_{2} d \mu_{1} d \mu_{2} d \nu_{1} d \nu_{2}$.

The formula for purity written in terms of measurable optical tomograms (using the expression of symplectic tomogram in terms of the optical tomogram) [28, 29] was used in the experiments [30] on homodyne detection of some photon states.

For the fidelity and purity of quantum states of real physical resonant circuit, we have the inequalities

$\begin{aligned} 0 & \leq \frac{1}{2 \pi} \int w_{1}\left(J_{1}, \mu_{1}, \nu_{1}\right) w_{2}\left(J_{2},-\mu_{1},-\nu_{1}\right) \exp \left(i\left(J_{1}+J_{2}\right)\right) d J_{1} d J_{2} d \mu_{1} d \mu_{2} d \nu_{1} d \nu_{2} \leq 1, \\ 0 & \leq \frac{1}{2 \pi} \int w\left(J_{1}, \mu_{1}, \nu_{1}\right) w\left(J_{2},-\mu_{1},-\nu_{1}\right) \exp \left(i\left(J_{1}+J_{2}\right)\right) d J_{1} d J_{2} d \mu_{1} d \mu_{2} d \nu_{1} d \nu_{2} \leq 1\end{aligned}$

The tomograms associated with the states of a real physical resonant circuit must satisfy the inequality, which is the nonnegativity condition of the density operator,

$$
\int w(J, \mu, \nu) \exp (i(J \hat{1}-\mu \hat{I}-\nu \hat{Q})) d J d \mu d \nu \geq 0 .
$$

The obtained expressions for fidelities and purities are given in terms of measurable tomographic probability distributions. They can be applied for checking the quantumness of the states in experiments which are analogs of the homodyne detection of photon states. The Josephson-junction realization of circuit QED can be used to study quantum properties of the circuits. 


\section{Parametric Josephson junction}

As an application of our model we discussed above, we consider such object as the Josephson junction. The Josephson junction at zero temperature is described by the Hamiltonian [31,32]

$$
\hat{H}=\frac{\hat{Q}^{2}}{2 C}+\frac{\hbar I_{c}(t)}{2 e}(1-\cos \hat{\phi})-\frac{\hbar I_{k}(t) \hat{\phi}}{2 e},
$$

where $C$ is the capacitance of the junction, $I_{c}$ is the critical current, $\hat{Q}$ is the charge operator, $\hat{\phi}$ is the phase operator, $I_{k}(t)$ is the external classical current, $e$ is the electron charge, and $\hbar$ is the Planck constant.

Now we consider the Josephson junction in the domain of small phases. Also we assume that the shot noise is smaller than the quantum fluctuations, $\delta Q \gg 2 e$. This means that we have the following condition for the critical current and capacity of the Josephson junction:

$$
I_{c} C \gg \frac{32 e^{3}}{\hbar} \sim 10^{-21} \Phi A
$$

Under the condition (13), the term $1-\cos \hat{\phi}$ in the Hamiltonian (12) can be replaced by the quadratic expression $\hat{\phi}^{2} / 2$. So instead of the Hamiltonian (12) we obtain the Hamiltonian of a driven resonance circuit

$$
\hat{H}=\frac{\hat{Q}^{2}}{2 C}+\frac{\hbar I_{c}(t) \hat{\phi}^{2}}{2 e}-\frac{\hbar I_{k}(t) \hat{\phi}}{2 e} .
$$

The commutation relation for the charge and phase operators reads

$$
[\hat{\phi}, \hat{Q}]=2 i e,
$$

that provides the following commutation relation for the current $\hat{I}=-I_{c} \hat{\phi}$ and voltage $\hat{V}=\hat{Q} / C$ operators:

$$
[\hat{I}, \hat{V}]=i \hbar \omega^{2}
$$

where

$$
\omega=\left(2 e I_{c} / \hbar C\right)^{1 / 2}
$$

is the plasma frequency of the junction. If the parameters (critical current or capacity)

of the junction are dependent on time, then the plasma frequency is also the function of time, and so the Josephson junction is acted by a parametric excitation. Below we assume such the units that provide all the variables like the current and voltage to be dimensionless. The Josephson-junction Gaussian state can be determined by the tomogram

$$
w(J, \mu, \nu, t)=\frac{1}{\sqrt{2 \pi \sigma_{J}(t)}} \exp \left(-\frac{(J-\bar{J})^{2}}{2 \sigma_{J}(t)}\right)
$$


where the dispersion of the observable is expressed through the dispersions of the current and voltage as follows:

$$
\sigma_{J}(t)=\mu^{2} \sigma_{I^{2}}(t)+\nu^{2} \sigma_{V^{2}}(t)+2 \mu \nu \sigma_{I V}(t)
$$

and the dispersions of the current and voltage are

$$
\sigma_{I^{2}}(t)=|\epsilon(t)|^{2} / 2, \quad \sigma_{V^{2}}(t)=|\dot{\epsilon}(t)|^{2} / 2, \quad \sigma_{I V}=\sqrt{\sigma_{I^{2}} \sigma_{V^{2}}-1 / 4}
$$

The function $\epsilon(t)$ in (20) satisfies the equation

$$
\ddot{\epsilon}(t)+\omega^{2}(t) \epsilon(t)=0
$$

with additional condition

$$
\dot{\epsilon} \epsilon^{*}-\dot{\epsilon}^{*} \epsilon=2 i
$$

where the time-dependent frequency in Eq. (21) is given by (17) with the time-dependent critical current. The mean values of observable $J$ is

$$
\bar{J}=-\sqrt{2}\left[\mu\left(\operatorname{Re}\left(\delta \epsilon^{*}\right)+\nu \operatorname{Re}\left(\delta \dot{\epsilon}^{*}\right)\right]\right.
$$

where function $\delta(t)$ is

$$
\delta(t)=-\frac{i}{\sqrt{2}} \int_{0}^{t} I_{k}(\tau) \epsilon(\tau) d \tau .
$$

In such a system, an analog of the well-known Casimir effect can exist. The Casimir force is an attraction force which exists between two noncharged plates without photons between them. The Casimir force exists due to the dependence of the electromagneticfield vacuum energy on the geometry of the system. The vacuum energy depends on the parameters of the system. This means, that the forces exist which try to minimize the vacuum energy. In the parametric case where the system has time-dependent parameters, the nonstationary Casimir effect appears. The energy of external mechanical source goes to reforming the vacuum energy, and the electromagnetic radiation appears [33]. In [6 10], it was suggested to use the parametric Josephson junction for obtaining an analog of the nonstationary Casimir effect. Due to nonstationary Casimir effect, there exists a possibility to obtain electric oscillations without connecting the junction with external electromagnetic sources due to changing the inductance or capacity of the junction. Applying the external energy, we change the critical current or capacity of the junction and obtain the energy of electric oscillations. So, the Josephson junction with varying parameters can be used as a quantum generator of current, as was suggested in [6 10].

Nowadays, the discussions of analogous effects are known as the circuit quantum electrodynamics. These effects were studied, e.g., in [11-13, 34, 35]. 


\section{Two interacting resonant circuits}

In this section, we obtain an explicit form of the two-mode symplectic tomogram of the ground quantum state for two interacting resonant circuits. We also obtain new tomographic characteristics for this state such as the tomographic entropy and information. We consider two high-quality interacting resonant circuits with inductances $L_{n}$ and capacities $C_{n}$. The Hamiltonian of the system is of the form

$$
\hat{H}=\frac{L \hat{I}_{1}^{2}}{2}+\frac{\hat{Q}_{1}^{2}}{2 C}+\frac{L \hat{I}_{1}^{2}}{2}+\frac{\hat{Q}_{1}^{2}}{2 C}+L_{12} \hat{I}_{1} \hat{I}_{2}
$$

where $\hat{Q}_{1}$ and $\hat{Q}_{2}$ are the operator of charge on plates of the capacitor in the first and second circuits, $\hat{I}_{1}$ and $\hat{I}_{2}$ are operators of current in the first and second circuits, respectively, and $L_{12}$ is the selfinductance. The commutation relations for operators of charges and currents read

$$
\left[\hat{I}_{1}, \hat{Q}_{1}\right]=i \hbar / L, \quad\left[\hat{I}_{2}, \hat{Q}_{2}\right]=i \hbar / L, \quad\left[\hat{I}_{1}, \hat{Q}_{2}\right]=\left[\hat{I}_{2}, \hat{Q}_{1}\right]=0 .
$$

For operators of voltage on the plates of capacitors $\hat{V}_{1}=\hat{Q}_{1} / C$ and $\hat{V}_{2}=\hat{Q}_{2} / C$, one has the commutation relations with operators of currents $\left[\hat{I}_{1}, \hat{V}_{1}\right]=\left[\hat{I}_{2}, \hat{V}_{2}\right]=i \hbar \omega^{2}$, where $\omega$ is the plasma frequency. Introducing the new variables

$$
\hat{I}_{k}=\left(\hat{I}_{1}+\hat{I}_{2}\right) / \sqrt{2}, \quad \hat{I}_{s}=\left(\hat{I}_{1}-\hat{I}_{2}\right) / \sqrt{2},
$$

we obtain the Hamiltonian (23) in the form

$$
\hat{H}=\frac{L_{k} \hat{I}_{k}^{2}}{2}+\frac{\hat{Q}_{k}^{2}}{2 C}+\frac{L_{s} \hat{I}_{s}^{2}}{2}+\frac{\hat{Q}_{s}^{2}}{2 C},
$$

where $L_{k}=L+L_{12}$ and $L_{s}=L-L_{12}$. The frequencies of oscillations of two noninteracting resonant circuits with the Hamiltonian (25) are $w_{s}=\omega \sqrt{L / L_{s}}$ and $w_{k}=\omega \sqrt{L / L_{k}}$, and new amplitudes of the vacuum fluctuations read

$$
i_{k}=\sqrt{\hbar \omega / L_{k}}, \quad q_{k}=\sqrt{\hbar / \omega L_{k}}, \quad i_{s}=\sqrt{\hbar \omega / L}, \quad q_{s}=\sqrt{\hbar / \omega L_{s}} .
$$

The system of two interacting high-quality resonant circuits can also be considered within the framework of symplectic-tomography scheme [15]. Below in this section and Appendix, we assume the current, charge, and voltage to be dimensionless. We introduce the general linear combinations of currents and charges as follows:

$$
\hat{J}_{1}=\mu_{1} \hat{I}_{1}+\nu_{1} \hat{Q}_{1}, \quad \hat{J}_{2}=\mu_{2} \hat{I}_{2}+\nu_{2} \hat{Q}_{2},
$$

where $\mu_{1}, \mu_{2}, \nu_{1}$, and $\nu_{2}$ are real numbers, which label a reference frame in the currentscharge space $\left(I_{1}, I_{2}, Q_{1}, Q_{2}\right)$ of the system. 
The state of the system of two resonant circuits can also be determined by symplectic tomogram, which is the function of observables $J_{1}$ and $J_{2}$ and additional variables $\mu_{1}$, $\mu_{2}, \nu_{1}$, and $\nu_{2}$. For example, the symplectic tomogram of the system Gaussian state is

$$
w\left(J_{1}, J_{2}, \mu_{1}, \mu_{2}, \nu_{1}, \nu_{2}\right)=\frac{1}{2 \pi \sqrt{\operatorname{det} \sigma(t)}} \exp \left(-\frac{1}{2} \mathbf{J} \sigma^{-\mathbf{1}}(\mathbf{t}) \mathbf{J}\right),
$$

where components of the vector $\mathbf{J}$ are observables $J_{1}$ and $J_{2}$ and the dispersion matrix $\sigma(t)$

$$
\sigma(t)=\left(\begin{array}{cc}
\sigma_{J_{1} J_{1}} & \sigma_{J_{1} J_{2}} \\
\sigma_{J_{1} J_{2}} & \sigma_{J_{2} J_{2}}
\end{array}\right)
$$

are expressed through the dispersion of current and charge in the form

$$
\begin{aligned}
& \sigma_{J_{1} J_{1}}=\mu_{1} \sigma_{I_{1}^{2}}(t)+\nu_{1} \sigma_{Q_{1}^{2}}(t)+2 \mu_{1} \nu_{1} \sigma_{I_{1} Q_{1}}(t) \\
& \sigma_{J_{2} J_{2}}=\mu_{2} \sigma_{I_{2}^{2}}(t)+\nu_{2} \sigma_{Q_{2}^{2}}(t)+2 \mu_{2} \nu_{2} \sigma_{I_{2} Q_{2}}(t) \\
& \sigma_{J_{1} J_{2}}=\mu_{1} \nu_{2} \sigma_{I_{1} Q_{2}}(t)+\mu_{2} \nu_{1} \sigma_{I_{2} Q_{1}}(t)+\mu_{1} \mu_{2} \sigma_{I_{1} I_{2}}(t)+\nu_{1} \nu_{2} \sigma_{Q_{1} Q_{2}}(t) .
\end{aligned}
$$

Explicit expressions for the current-charge dispersion-matrix elements are given in Appendix by formulae (31)-(34).

Due to the fact that the symplectic tomogram is the probability distribution function, following the Shannon prescription, we can associate the tomographic entropy [16] with the state of two interacting circuits described by the tomogram in the probability representation of quantum mechanics as follows:

$$
S\left(\mu_{1}, \mu_{2}, \nu_{1}, \nu_{2}\right)=-\int w\left(J_{1}, J_{2}, \mu_{1}, \mu_{2}, \nu_{1}, \nu_{2}\right) \ln w\left(J_{1}, J_{2}, \mu_{1}, \mu_{2}, \nu_{1}, \nu_{2}\right) d J_{1} d J_{2} .
$$

Also we can introduce the tomographic information associates with the system state

$$
\mathcal{I}=\int w\left(J_{1}, J_{2}, \mu_{1}, \mu_{2}, \nu_{1}, \nu_{2}\right) \ln \left[\frac{w\left(J_{1}, J_{2}, \mu_{1}, \mu_{2}, \nu_{1}, \nu_{2}\right)}{w_{1}\left(J_{1}, \mu_{1}, \nu_{1}\right) w_{2}\left(J_{2}, \mu_{2}, \nu_{2}\right)}\right] d J_{1} d J_{2}
$$

where

$$
\begin{aligned}
& w_{1}\left(J_{1}, \mu_{1}, \nu_{1},\right)=\int w\left(J_{1}, J_{2}, \mu_{1}, \mu_{2}, \nu_{1}, \nu_{2}\right) d J_{2}, \\
& w_{2}\left(J_{2}, \mu_{2}, \nu_{2}\right)=\int w\left(J_{1}, J_{2}, \mu_{1}, \mu_{2}, \nu_{1}, \nu_{2}\right) d J_{1} .
\end{aligned}
$$

Thus, we got two tomographic characteristics of two circuit states describing the degree of quantum correlations in the system state, such as entropy and information. 


\section{Conclusions}

Concluding, we point out the main results of our study.

We reviewed the notion of quantum state in the symplectic tomography approach on the examples of two interacting high-quality circuits and Josephson junctions and discussed an analog of the nonstationary Casimir effect in the Josephson junction. We presented the entropy, information, fidelity, and purity of the circuit states in the tomographic-probability representation. For the Gaussian state of the resonant circuit, we introduced the tomographic entropy and expressed the entropy in terms of the symplectic tomogram of the resonant-circuit state. For two different quantum resonant circuit states, we obtained the expression for fidelity in terms of symplectic tomograms of these states. Particulary, we got the expression for purity of the resonant circuit state in terms of symplectic tomograms. We got inequalities which determine the quantumness of the circuit states. For the system of two high-quality interacting resonant circuits, we introduced the tomographic entropy and tomographic information.

\section{Acknowledgments}

The study was supported by the Russian Foundation for Basic Research under Project No. 10-02-00312. The author is grateful to the Organizers of the 19th Central European

Workshop on Quantum Optics (Sinaia, Romania, 2-6 July 2012) and especially Prof. Aurelian Isar for invitation and kind hospitality.

\section{Appendix}

Assuming dimensionless variables $\omega_{k}$ and $\omega_{s}$ and $\omega=1$, we calculate matrix elements of the dispersion matrix

$$
\begin{aligned}
& \sigma_{Q_{1}^{2}}(t)=\frac{1}{4}\left[c_{+}^{2} \sigma_{Q_{1}^{2}}(0)+c_{-}^{2} \sigma_{Q_{2}^{2}}(0)+k_{+}^{2} \sigma_{I_{1}^{2}}(0)+k_{-}^{2} \sigma_{I_{2}^{2}}(0)+2\left(k_{+} k_{-} \sigma_{I_{1} I_{2}}(0)+c_{+} k_{+} \sigma_{I_{1} Q_{1}}(0)\right.\right. \\
& \left.\left.+c_{+} c_{-} \sigma_{Q_{1} Q_{2}}(0)+k_{-} c_{-} \sigma_{I_{2} Q_{2}}(0)+k_{-} c_{-} \sigma_{Q_{1} I_{2}}(0)+k_{+} c_{-} \sigma_{I_{1} Q_{2}}(0)\right)\right] \\
& \sigma_{Q_{2}^{2}}(t)=\frac{1}{4}\left[c_{-}^{2} \sigma_{Q_{1}^{2}}(0)+c_{+}^{2} \sigma_{Q_{2}^{2}}(0)+k_{-}^{2} \sigma_{I_{1}^{2}}(0)+k_{+}^{2} \sigma_{I_{2}^{2}}(0)+2\left(k_{+} k_{-} \sigma_{I_{1} I_{2}}(0)+c_{-} k_{-} \sigma_{I_{1} Q_{1}}(0)\right.\right. \\
& \left.\left.+c_{+} c_{-} \sigma_{Q_{1} Q_{2}}(0)+k_{-} c_{+} \sigma_{I_{1} Q_{2}}(0)+k_{+} c_{-} \sigma_{Q_{1} I_{2}}(0)-k_{+} c_{+} \sigma_{I_{2} Q_{2}}(0)\right)\right] \\
& \sigma_{Q_{1} Q_{2}}(t)=\frac{1}{4}\left[c_{-} c_{+}\left(\sigma_{Q_{1}^{2}}(0)+\sigma_{Q_{2}^{2}}(0)\right)+k_{-} k_{+}\left(\sigma_{I_{1}^{2}}(0)+\sigma_{I_{2}^{2}}(0)\right)+\left(k_{+}^{2}+k_{-}^{2}\right) \sigma_{I_{1} I_{2}}(0)\right. \\
& +\left(c_{-} k_{+}+k_{-} c_{+}\right)\left(\sigma_{I_{1} Q_{1}}(0)+\sigma_{I_{2} Q_{2}}(0)\right)+\left(c_{+}^{2}+c_{-}^{2}\right) \sigma_{Q_{1} Q_{2}}(0) \\
& \left.+\left(k_{-} c_{-}+k_{+} c_{+}\right)\left(\sigma_{I_{1} Q_{2}}(0)+\sigma_{Q_{1} I_{2}}(0)\right)\right] .
\end{aligned}
$$


Fidelity and purity of quantum electrical circuit states

The dispersions of currents after interaction read

$$
\begin{aligned}
& \sigma_{I_{1}^{2}}(t)=\frac{1}{4}\left[s_{+}^{2} \sigma_{Q_{1}^{2}}(0)+s_{-}^{2} \sigma_{Q_{2}^{2}}(0)+c_{+}^{2} \sigma_{I_{1}^{2}}(0)+c_{-}^{2} \sigma_{I_{2}^{2}}(0)+2\left(c_{+} c_{-} \sigma_{I_{1} I_{2}}(0)-c_{+} s_{+} \sigma_{I_{1} Q_{1}}(0)\right.\right. \\
& \left.\left.+s_{+} s_{-} \sigma_{Q_{1} Q_{2}}(0)-s_{-} c_{-} \sigma_{I_{2} Q_{2}}(0)-s_{+} c_{-} \sigma_{Q_{1} I_{2}}(0)-c_{+} s_{-} \sigma_{I_{1} Q_{2}}(0)\right)\right], \\
& \sigma_{I_{2}^{2}}(t)=\frac{1}{4}\left[s_{-}^{2} \sigma_{Q_{1}^{2}}(0)+s_{+}^{2} \sigma_{Q_{2}^{2}}(0)+c_{-}^{2} \sigma_{I_{1}^{2}}(0)+c_{+}^{2} \sigma_{I_{2}^{2}}(0)+2\left(c_{+} c_{-} \sigma_{I_{1} I_{2}}(0)-c_{-} s_{-} \sigma_{I_{1} Q_{1}}(0)\right.\right. \\
& \left.\left.+s_{+} s_{-} \sigma_{Q_{1} Q_{2}}(0)-c_{-} s_{+} \sigma_{I_{1} Q_{2}}(0)-s_{-} c_{+} \sigma_{Q_{1} I_{2}}(0)-s_{+} c_{+} \sigma_{I_{2} Q_{2}}(0)\right)\right], \\
& \sigma_{I_{1} I_{2}}(t)=\frac{1}{4}\left[s_{-} s_{+}\left(\sigma_{Q_{1}^{2}}(0)+\sigma_{Q_{2}^{2}}(0)\right)+c_{-} c_{+}\left(\sigma_{I_{1}^{2}}(0)+\sigma_{I_{2}^{2}}(0)\right)+\left(c_{+}^{2}+c_{-}^{2}\right) \sigma_{I_{1} I_{2}}(0)\right. \\
& -\left(c_{-} s_{+}+s_{-} c_{+}\right)\left(\sigma_{I_{1} Q_{1}}(0)+\sigma_{I_{2} Q_{2}}(0)\right)+\left(s_{+}^{2}+s_{-}^{2}\right) \sigma_{Q_{1} Q_{2}}(0) \\
& \left.+\left(s_{-} c_{-}+s_{+} c_{+}\right)\left(\sigma_{I_{1} Q_{2}}(0)+\sigma_{Q_{1} I_{2}}(0)\right)\right] .
\end{aligned}
$$

The covariances are

$$
\begin{aligned}
& \sigma_{I_{1} Q_{1}}(t)=\frac{1}{4}\left[-c_{+} s_{+} \sigma_{Q_{1}^{2}}(0)-s_{-} c_{-} \sigma_{Q_{2}^{2}}(0)+c_{+} k_{+} \sigma_{I_{1}^{2}}(0)+c_{-} k_{-} \sigma_{I_{2}^{2}}(0)\right. \\
& -\left(c_{-} s_{+}+s_{-} c_{+}\right) \sigma_{Q_{1} Q_{2}}(0)+\left(c_{-} k_{+}+c_{+} k_{-}\right) \sigma_{I_{1} I_{2}}(0)+\left(c_{+}^{2}-k_{+} s_{+}\right) \sigma_{I_{1} Q_{1}}(0) \\
& \left.+\left(c_{-}^{2}-s_{-} k_{-}\right) \sigma_{I_{2} Q_{2}}(0)+\left(c_{+} c_{-}-k_{-} s_{+}\right) \sigma_{Q_{1} I_{2}}(0)+\left(c_{+} c_{-}-s_{-} k_{+}\right) \sigma_{I_{1} Q_{2}}(0)\right] \\
& \sigma_{I_{2} Q_{2}}(t)=\frac{1}{4}\left[-c_{-} s_{-} \sigma_{Q_{1}^{2}}(0)-s_{+} c_{+} \sigma_{Q_{2}^{2}}(0)+c_{-} k_{-} \sigma_{I_{1}^{2}}(0)+c_{+} k_{+} \sigma_{I_{2}^{2}}(0)\right. \\
& -\left(c_{+} s_{-}+s_{+} c_{-}\right) \sigma_{Q_{1} Q_{2}}(0)+\left(c_{-} k_{+}+c_{+} k_{-}\right) \sigma_{I_{1} I_{2}}(0)+\left(c_{-}^{2}-k_{-} s_{-}\right) \sigma_{I_{1} Q_{1}}(0) \\
& \left.+\left(c_{+}^{2}-s_{+} k_{+}\right) \sigma_{I_{2} Q_{2}}(0)+\left(c_{+} c_{-}-k_{+} s_{-}\right) \sigma_{Q_{1} I_{2}}(0)+\left(c_{+} c_{-}-s_{+} k_{-}\right) \sigma_{I_{1} Q_{2}}(0)\right] \\
& \sigma_{I_{2} Q_{1}}(t)=\frac{1}{4}\left[-c_{+} s_{-} \sigma_{Q_{1}^{2}}(0)-s_{+} c_{-} \sigma_{Q_{2}^{2}}(0)+c_{-} k_{+} \sigma_{I_{1}^{2}}(0)+c_{+} k_{-} \sigma_{I_{2}^{2}}(0)\right. \\
& -\left(c_{+} s_{+}+s_{-} c_{-}\right) \sigma_{Q_{1} Q_{2}}(0)+\left(c_{+} k_{+}+c_{-} k_{-}\right) \sigma_{I_{1} I_{2}}(0)+\left(c_{+} c_{-}-k_{+} s_{-}\right) \sigma_{I_{1} Q_{1}}(0) \\
& \left.+\left(c_{+} c_{-}-s_{+} k_{-}\right) \sigma_{I_{2} Q_{2}}(0)+\left(c_{+}^{2}-k_{-} s_{-}\right) \sigma_{Q_{1} I_{2}}(0)+\left(c_{-}^{2}-s_{+} k_{+}\right) \sigma_{I_{1} Q_{2}}(0)\right] \\
& \sigma_{I_{1} Q_{2}}(t)=\frac{1}{4}\left[-c_{-} s_{+} \sigma_{Q_{1}^{2}}(0)-s_{-} c_{+} \sigma_{Q_{2}^{2}}(0)+c_{+} k_{-} \sigma_{I_{1}^{2}}(0)+c_{-} k_{+} \sigma_{I_{2}^{2}}(0)\right. \\
& -\left(c_{+} s_{+}+s_{-} c_{-}\right) \sigma_{Q_{1} Q_{2}}(0)+\left(c_{+} k_{+}+c_{-} k_{-}\right) \sigma_{I_{1} I_{2}}(0)+\left(c_{+} c_{-}-k_{-} s_{+}\right) \sigma_{I_{1} Q_{1}}(0) \\
& \left.+\left(c_{+} c_{-}-s_{-} k_{+}\right) \sigma_{I_{2} Q_{2}}(0)+\left(c_{-}^{2}-k_{+} s_{+}\right) \sigma_{Q_{1} I_{2}}(0)+\left(c_{+}^{2}-s_{-} k_{-}\right) \sigma_{I_{1} Q_{2}}(0)\right]
\end{aligned}
$$

where

$c_{ \pm}=\cos \omega_{k} t \pm \cos \omega_{s} t, \quad k_{ \pm}=\frac{\sin \omega_{k} t}{\omega_{k}} \pm \frac{\sin \omega_{s} t}{\omega_{s}}, \quad s_{ \pm}=\sin \omega_{k} t \omega_{k} \pm \sin \omega_{s} t \omega_{s}$

\section{References}

[1] Dodonov V V 2010 Phys. Scr. 82038105

[2] Dodonov V V and Dodonov A V 2005 J. Russ. Laser Res. 26445

[3] Dodonov A V, Dodonov E V and Dodonov V V 2003 Phys. Lett. A 317378 
[4] Wilson C M, Johansson G, Pourkabirian A, Simoen M, Johansson J R, Duty T, Nori F and Delsing P 2011 Nature 479 Issue: 7373

[5] Dodonov V V, Man'ko V I and Man'ko O V 1991 Correlated States and Gravitational Waveguide in: Proceedings of the Lebedev Physical Institute Vol. 200, p. 155 (Moscow: Nauka) [tr: New York, Commack: Nova Science]

[6] Dodonov V V, Man'ko V I and Man'ko O V 1989 J. Sov. Laser Res. 10413 (New York: Plenum)

[7] Dodonov V V, Man'ko V I and Man'ko O V 1992 J. Sov. Laser Res. 13196 (New York: Plenum)

[8] Dodonov V V, Man'ko V I and Man'ko O V 1990 Measurement Techniq. USSR 33102

[9] Man'ko V I 1991 J. Sov. Laser Res. 12383 (New York: Plenum)

[10] Man’ko O V 1994 J. Korean Phys. Soc. 271

[11] Takashima K, Hatakenaka N, Kurihara S and Zeilinger A 2008 J. Phys. A: Math. Theor. 41164036

[12] Takashima K, Matsuo S, Fujii T, Hatakenaka N, Kurihara S and Zeilinger A 2009 J. Phys.: Conf. Ser. 150052260

[13] Fujii T, Matsuo S, Hatakenaka N, Kurihara S and Zeilinger A 2011 Phys. Rev. B 84521

[14] Ibort A, Man'ko V I, Marmo G, Simoni A and Ventriglia F 2009 Phys. Scr. 79065013

[15] Mancini S, Man'ko V I and Tombesi P 1996 Phys. Lett. A 2131

[16] Man'ko O V and Man'ko V I 1997 J. Russ. Laser Res. 18407

[17] Man'ko M A 2001 J. Russ. Laser Res. 22168

[18] Man'ko M A and Man'ko V I 2011 Found Phys. 41330

[19] Man'ko O V 2012 Quantum Tomography of Current and Voltage States in Nanoelectric Circuits in: Proceedings of the International Conference on Foundations of Probability and Physics, FPP-6, Växjö, Sweeden, 2011 [AIP Conf. Proc. 1424 221]

[20] Wigner E 1932 Phys. Rev. 40749

[21] Bertrand J and Bertrand P 1989 Found. Phys. 17397

[22] Smithey D T, Beck M, Raymer M G and Faridani A 1993 Phys. Rev. Lett. 701244

[23] D'Ariano G M, Mancini S, Man'ko V I and Tombesi P 1996 J. Opt. B: Quantum Semiclass. Opt. 81017

[24] De Nicola S, Fedele R, Man'ko M A and Man'ko V I 2003 Eur. Phys. J. B 36385

[25] Man'ko O V and Man'ko V I 2004 J. Russ. Laser Res. 25115

[26] Man'ko O V 2007 J. Russ. Laser Res. 28125

[27] Man'ko O V and Tcherniega N V 2006 Proc. SPIE 6256625

[28] Man'ko O V and Man'ko V I 2009 Fortschr. Phys. 571064

[29] Man'ko V I, Marmo G, Porzio A, Solimeno S and Ventriglia F 2011 Phys. Scr. 83045001

[30] Bellini M, Coelho A S, Fillipov S N, Man'ko V I and Zavatta A 2012 Phys. Rev. A 85052129

[31] Anderson P W 1964 Many Body Problem (New York: Academic Press) p. 113

[32] Likharev K K 1985 Introduction to the Dynamics of Josephson Junction (Moscow: Nauka) [in Russian]

[33] Dodonov V V, Man'ko V I and Man'ko O V 1992 in: Proceedings of the Lebedev Physical Institute Vol. 208, p. 174 (Moscow: Nauka) [tr: New York, Commack: Nova Science]

[34] Mallet F, Castellanos-Beltran M A, Ku H S, Irwin K D, Hilton G C, Vale L R and Lehnert K W 2011 Phys. Rev. Lett. 106220502

[35] Eichier C, Bozyigit D, Lang C, Steffen L, Fink J and Walraff A 2011 Phys. Rev. Lett., 106, 220503 